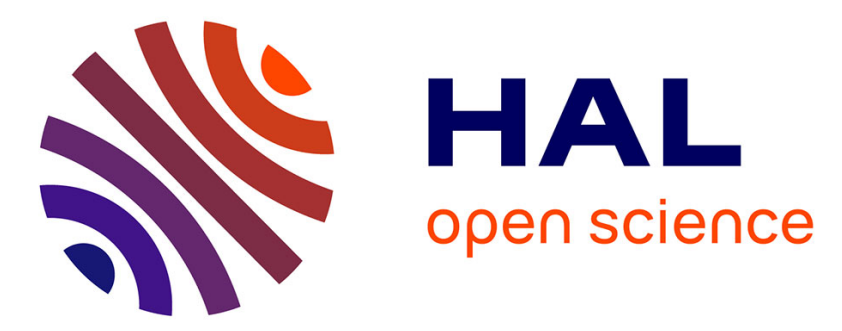

\title{
Implementation of Scale Invariant Feature Transform detector on FPGA for low-power wearable devices for prostheses control
}

Attila Fejér, Zoltán Nagy, Jenny Benois-pineau, Péter Szolgay, Aymar de Rugy, Jean-philippe Domenger

\section{To cite this version:}

Attila Fejér, Zoltán Nagy, Jenny Benois-pineau, Péter Szolgay, Aymar de Rugy, et al.. Implementation of Scale Invariant Feature Transform detector on FPGA for low-power wearable devices for prostheses control. International Journal of Circuit Theory and Applications, 2021, 49, pp.2255 2273. 10.1002/cta.3025 . hal-03373309

\section{HAL Id: hal-03373309 \\ https://hal.science/hal-03373309}

Submitted on 11 Oct 2021

HAL is a multi-disciplinary open access archive for the deposit and dissemination of scientific research documents, whether they are published or not. The documents may come from teaching and research institutions in France or abroad, or from public or private research centers.
L'archive ouverte pluridisciplinaire HAL, est destinée au dépôt et à la diffusion de documents scientifiques de niveau recherche, publiés ou non, émanant des établissements d'enseignement et de recherche français ou étrangers, des laboratoires publics ou privés. 


\title{
Implementation of Scale Invariant Feature Transform detector on FPGA for low-power wearable devices for prostheses control
}

\author{
Attila Fejér ${ }^{1,2}$ (1) | Zoltán Nagy² (i) | Jenny Benois-Pineau ${ }^{1}$ | Péter Szolgay ${ }^{2}$ | \\ Aymar de Rugy $^{3}$ | Jean-Philippe Domenger ${ }^{1}$
}

\author{
${ }^{1}$ Laboratoire Bordelais de Recherche en \\ Informatique, University of Bordeaux, \\ Bordeaux, France \\ ${ }^{2}$ Faculty of Information Technology and \\ Bionics, Pázmány Péter Catholic \\ University, Budapest, Hungary \\ ${ }^{3}$ Institut de Neurosciences Cognitives et \\ Intégratives d'Aquitaine, University of \\ Bordeaux, Bordeaux, France

\section{Correspondence} \\ Attila Fejér, Laboratoire Bordelais de \\ Recherche en Informatique, University of \\ Bordeaux, Bordeaux, France, and Faculty \\ of Information Technology and Bionics, \\ Pázmány Péter Catholic University, \\ Budapest, Hungary. \\ Email: fejer.attila@itk.ppke.hu

\section{Funding information} \\ Széchenyi 2020 Program; Human \\ Resource Development Operational \\ Program; Program of Integrated Territorial \\ Investments in Central-Hungary, Grant/ \\ Award Numbers: EFOP- \\ 3.6.2-16-2017-00013, 3.6.3-vekop- \\ 16-2017-00002; European Structural and \\ Investment; CNRS; Balaton PHC
}

\begin{abstract}
Summary
In this paper we describe an FPGA implementation of the Scale Invariant Feature Transform (SIFT) algorithm. The FPGA is required as its a lightweight device which makes it ideal for vision-guided hybrid neuro-prostheses utilised for upper limbs replacement. SIFT point detection is needed for computation of coordinates of the object-to-grasp in a wearable multi-camera system. A modified SIFT algorithm is proposed and an implementation of it into $\mathrm{C} / \mathrm{C}++$ language on Xilinx ZCU102 FPGA board. The proposed hybrid hardware/ software solution is compared to other hardware or hybrid implementations of the SIFT algorithm and with the baseline software detector OpenSIFT. The algorithm optimised for FPGA gives an average precision of 0.84 and the average recall of 0.94 in SIFT-point detection compared to the baseline.

The proposed solution has lower dissipated power than other solutions like CPU or GPU, and it has better computational speed.

This solution allows for processing medium-sized images in real-time with low power consumption.

\section{K E Y W O R D S}

computer vision, FPGA, image processing, prosthetic arm, SIFT
\end{abstract}

\section{1 | INTRODUCTION}

In assistive robotics research quite a strong effort is being allocated to the design of vision-guided neuro-prostheses. ${ }^{1}$ Using such a perceptual channel as vision allows to avoid discomfort related to the introduction of electrodes into remaining muscle in order to control the prosthesis. ${ }^{2}$ In the scenario we proposed ${ }^{1}$ a human subject is instrumented with a glass-worn eye-tracker with a scene camera (Tobii Pro glasses, for instance) and a RGB-depth or stereo camera mounted on the prosthesis. Computer vision software assists the person in object-to-grasp detection and localization using the glass-worn camera and then the software localises the object in the prosthesis-mounted camera view. For the 
latter matching of Scale Invariant Feature Transform (SIFT) points from different views has been proposed. ${ }^{3}$ The block diagram of the whole Hardware-Software (HW-SW) system and the illustration of the scenario is shown in Figure 1. In the object detection module the object which the person intends to grasp is recognised and localised in Tobii video frame by Convolutional Neural Network (CNN) classifier. Then the object-of-interest has to be found in prosthesis mounted camera's frames (the red block in Figure 1). For this the SIFT points are detected in the glass-view video frames in the object area and prosthesis-mounted camera view. The Homography matrix is computed by matching SIFT $^{4}$ points and homography parameters estimation with Random sample consensus (RANSAC) estimator. Then the object is projected into prosthesis-mounted camera view and the depth map is extracted for prosthesis servoing. The illustration of SIFT points matching between two views is presented in Figure 2. The algorithmic chain of the proposed scenario is being validated, the aim of this research is to develop a wearable device for control of a prosthetic arm. Hence, the SIFT detector implementation on FPGA is a part of the whole hybrid HW-SW system.

The contribution of this paper is two-fold.

- A modified SIFT detector approach is proposed that is more adapted to the FPGA implementation. A hardware/ software solution is developed to partition the system into a programmable logic and an embedded microprocessor;

- The SIFT detector is implemented on FPGA and three solutions is being compared: a software detector, our implementation on FPGA, GPU and analog VLSI implementation.

The remaining part of the paper is organised as following: in Section 2 we present the related work on SIFT points detection which includes Hardware/Software, Software, or Hardware implementations. In Section 3 the theoretical background of the SIFT point detector is revised. The proposed implementation of the algorithm is presented in

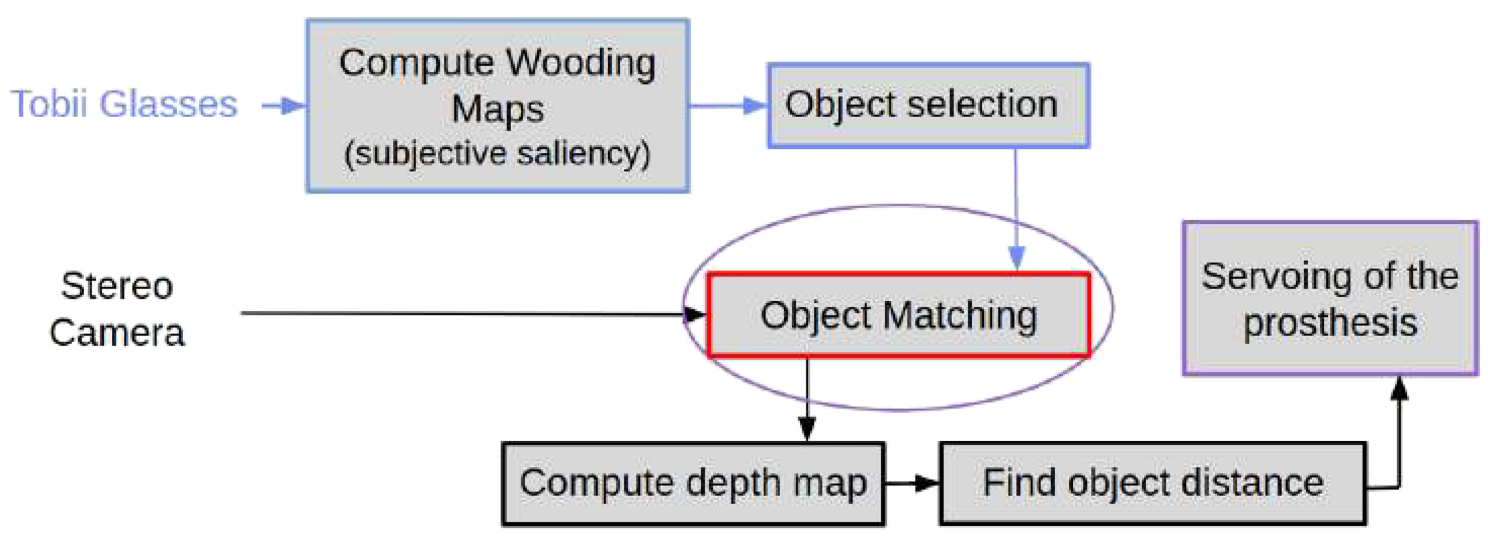

F I G U R E 1 Block diagram of the system vision assisted prosthetic system ${ }^{5}$ [Colour figure can be viewed at wileyonlinelibrary.com]

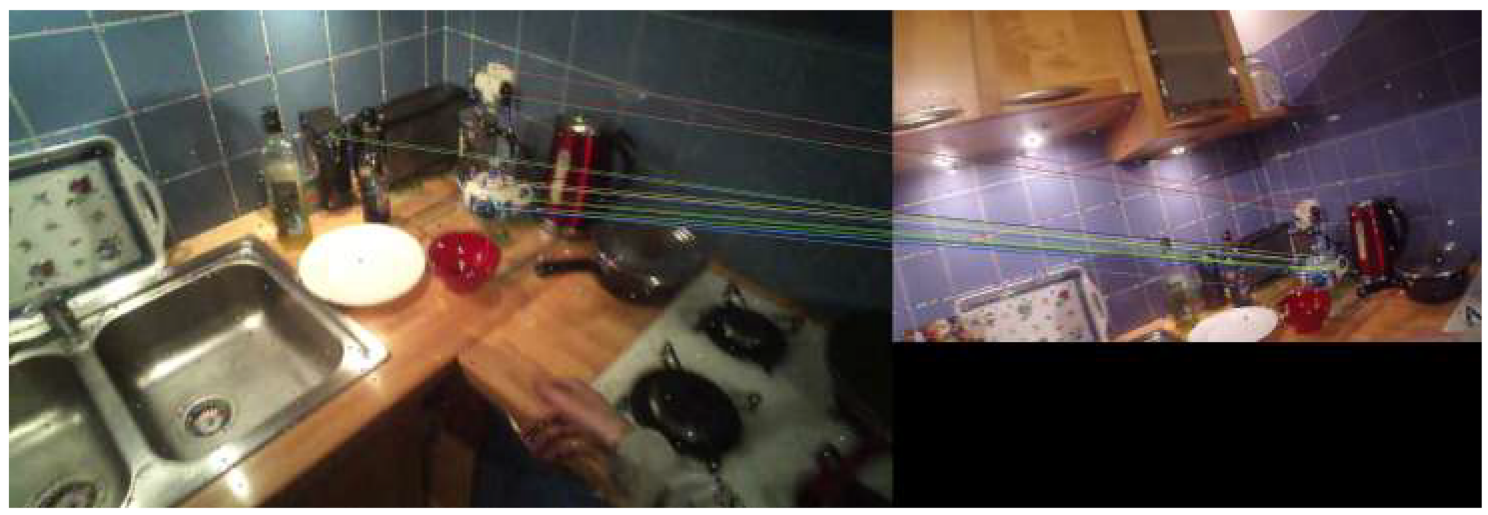

F I G U R E 2 Two different image Scale Invariant Feature Transform (SIFT) descriptor matches [Colour figure can be viewed at wileyonlinelibrary.com] 
Section 4 including our modified method, specifically adapted to the FPGA implementation. Experiments and results are given in Section 5. Finally, Section 6 concludes our work and outlines its perspectives.

\section{2 | RELATED WORK}

Computing power is a multi-parameter vector: any algorithm solving a problem will have a speed-power-area-bandwidth-accuracy metric. In the follow-up of this section a review of available solutions is being presented based on this multi-criteria point of view.

There are several keypoint detectors existing in computer vision such as Oriented FAST and Rotated BRIEF (ORB), ${ }^{6}$ Speeded-Up Robust Features (SURF), ${ }^{7}$ and the most widely used is SIFT. ${ }^{4}$ The previously mentioned detectors differ in the following way one of which is the technique by keypoints are extracted and also by the process the descriptors are computed and matched. ORB uses two main steps: an oriented Features computation from Accelerated Segments Test (FAST) ${ }^{8}$ which extracts the keypoints location and orientations of the keypoint combined with Binary robust independent elementary feature (BRIEF). ${ }^{9}$ The latter calculates the binary descriptors around extracted points. The $\mathrm{SURF}^{7}$ uses integral images and box filtering for extracting the keypoints and descriptors computation. Both $\mathrm{SURF}^{7}$ and $\mathrm{ORB}^{6}$ require less computational time than the SIFT. ${ }^{4}$ Nevertheless, we have chosen the SIFT algorithm to implement on FPGA because Karami et al. ${ }^{10}$ showed that in different kinds of transformations and deformations such as scaling, rotation, noise, fish-eye distortion and shearing SIFT outperformed other methods in precision.

SIFT is a widely used and implemented keypoint detector. There are CPU, ${ }^{11,12}$ GPU $^{13,14}$ and FPGA implementations. ${ }^{15-20}$ The OpenCV SIFT library ${ }^{11}$ and the OpenSIFT ${ }^{12}$ are popular frameworks for SIFT keypoint extraction and descriptor computation in CPUs. This is partially due to their flexibility as the input image resolution can be modified easily. However, the runtime of these CPU implementations are too slow for real-time image processing on light-weight devices. Computation can be accelereted by using GPUs, for example: CudaSIFT ${ }^{14}$ can process a 1280px $\times$ 960px image in $12.7 \mathrm{~ms}$, that is, 78.74 frames per second (fps) on the NVIDIA GeForce GTX 580 GPU. However, its power consumption is $244 \mathrm{~W}$, which is too high for a wearable application. HartSIFT ${ }^{13}$ can extract features within 3.14-10.57 ms (94.61-318 fps) depending on the input image size on the NVIDIA GeForce GTX TITAN Black. The power consumption of this GPU is $250 \mathrm{~W}$, which is also undesirably high for a portable device.

The most computationally intensive operation in SIFT keypoint extraction is the computation of the Gaussian pyramids as it requires multiplication of coefficients of Gaussian filters with scale-space images. For this step, an analog solution is developed by Rodríguez-Vázquez et al. ${ }^{21,22}$ where the Gaussian pyramid is computed by an analog CMOS circuit and exhibits very low dissipated power. One of the advantage of the inherent parallel processing is the high computational power of the analog VLSI implementations. However, the size of the array is small. ${ }^{21-23}$ In Suárez et al. ${ }^{22}$ the analog sensor/processor implementation has $88 \times 60$ processing elements, and each processing element has four photodiodes. The computation unit is connected to the vision sensor unit of the camera. The vision sensor array has $176 \times 120$ pixels only and the system is implemented using a $0.18 \mu \mathrm{m}$ CMOS technology. This solution is satisfactory from a computational point of view; however, it is not flexible with regard to increasing the video resolutions. The resolution of the analog sensor is not sufficiently high for our application, since our method use larger areas in video frames and it needs $480 \mathrm{px} \times 480 \mathrm{px}$ image as input.

Several different SIFT implementations on FPGA are published such as the system designed by Doménech-Asensi et $\mathrm{al},{ }^{15}$ which is a simplified version of the algorithm. It is assumed that each feature point has two main orientations at most, the orientation histogram uses 8 bins instead of 36 bins. Thus complexity is reduced but precision of orientations drops with regard to the original SIFT. ${ }^{4}$ The system can process $640 \mathrm{px} \times 480 \mathrm{px}$ sized input images at 99 fps processing speed on Xilinx Virtex-5, and it uses fixed point representation.

Rubio-Ibáñez et al. ${ }^{16}$ implemented the subpixel refinement stage of the SIFT algorithm. They used a ZedBoard (Xilinx Zynq7020) FPGA board and the rest of the algorithm was computed on the CPU.

Vourvoulakis et al. ${ }^{17,18}$ implemented an FPGA accelerated SIFT matching with RANSAC support. The architecture includes one octave and four scales. The Gaussian kernel computation is optimised to reduce the logic and memory resource requirements on the FPGA. Size of the circuit is further reduced by using 14bit fixed-point numbers during the Gaussian filter computation. In order to evaluate the first derivatives of the Difference of Gaussian (DoG) images Prewitt mask has been used. This step is required to remove the keypoints on the edges. Their system was implemented using VHDL on an Intel DE2i-150 board and can process 640px $\times 480 \mathrm{px}$ input video at $81 \mathrm{fps}$. 
Chang et al. ${ }^{19}$ proposed an architecture, where the Scale-space extrema are calculated on the FPGA and the rest of the algorithm on the CPU. The scale-space extrema detection uses separable kernel, which requires reduced logic and memory resource usage on the FPGA. Their implementation runs in a Xilinx Virtex II Pro FPGA, with a configuration of three octaves and six scales, and with a $145-\mathrm{MHz}$ clock frequency. An image of $320 \mathrm{px} \times 240 \mathrm{px}$ is processed in $1.1 \mathrm{~ms}$ (900 fps).

Shao et al. ${ }^{20}$ implemented the SIFT algorithm on a Virtex-5 FPGA borad, the input image has $292 \mathrm{px} \times 520$ px resolution. The system can process images with 38 fps. They changed the Gaussian pyramid building, instead of filtering in parallel with different size filters they cascaded several smaller size Gaussian kernels. The design of system is simplified, but requires more memory resources.

There also exists an FPGA implementation which handles the descriptor matching part after the keypoint extraction. ${ }^{24}$ The architecture is fully pipelined and uses 16-bit fixed-point number representation.

Hence, the efficient SIFT implementation on FPGA still remains an open research question. In this paper we propose an FPGA solution which is developed in a high-level language. Therefore, it is easier to modify some parameters such as the number of Gaussian layers compared to a VHDL or Verilog based SIFT implementations.

\section{SIFT}

SIFT method was proposed by Lowe ${ }^{4}$ and originally developed for image stitching to solve the problem in rotation-, scaling-, affine deformation-, viewpoint change-invariant way, remaining robust to noise and illumination changes when different images of the same scene are matched. For this reason it is particularly applicable for the of matching video frames coming from different acquisition devices with different scales and viewpoints of the same scene.

The SIFT algorithm has two main steps: (i) the keypoint detection and the (ii) descriptor computation. The keypoints are extracted from images to match first. Then invariant descriptors are computed in the vicinity of detected points and the matching process consists in comparison of descriptors. Keypoint detection and descriptor computation comprise several sub-steps which are depicted in Figure 3. Those steps are as follows:

A; Scale Space Extrema (SSE) Detection

B; Keypoint Localization

C; Orientation Assignment

D; Descriptor Generation

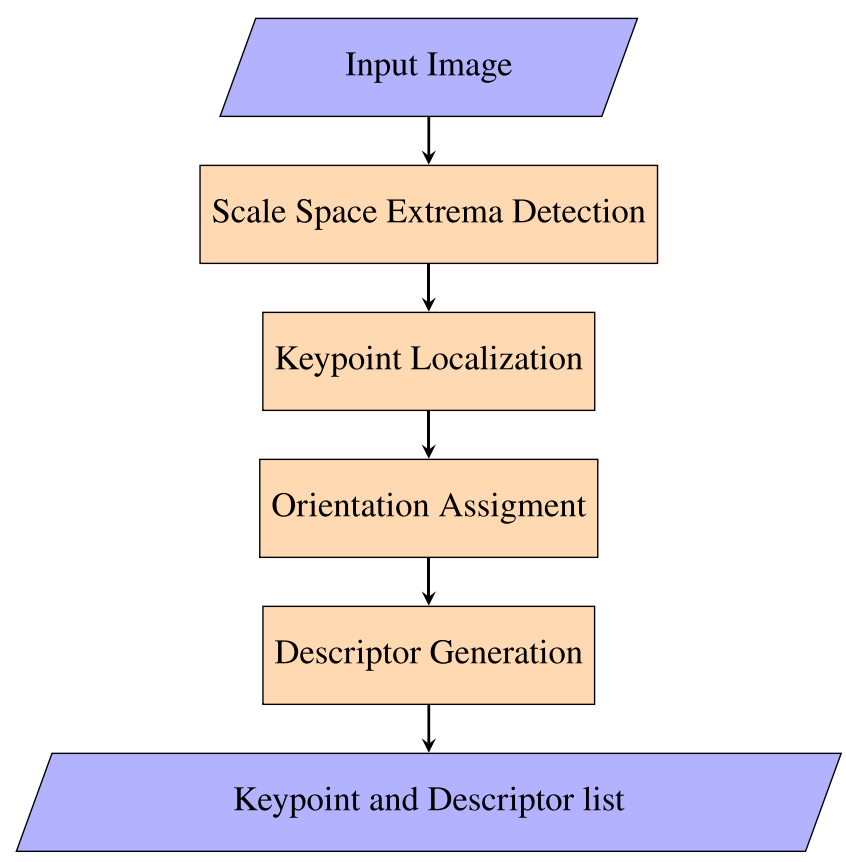

F I G U RE 3 The main steps of the Scale Invariant Feature Transform (SIFT) ${ }^{4}$ algorithm [Colour figure can be viewed at wileyonlinelibrary.com] 
In this paper we propose an FPGA implementation of SIFT point detection. Hence the next subsections contain the detailed description of the SIFT detection steps.

\section{1 | SSE detection}

The Input Image denoted by $I(x, y)$ is convolved by a Gaussian kernel $G(x, y, \sigma)$ resulting in the image of the scale-space $L(x, y, \sigma)$.

$$
L(x, y, \sigma)=G(x, y, \sigma) * I(x, y)
$$

where * is the convolution operator in $x$ and $y$, and $G(x, y, \sigma)$ is expressed by the following equation:

$$
G(x, y, \sigma)=\frac{1}{2 \pi \sigma^{2}} e^{\frac{-\left(x^{2}+y^{2}\right)}{2 \sigma^{2}}}
$$

Here $\sigma$ is the scale parameter. The next step is to detect candidate keypoint locations as the scale-space extrema in the DoG functions denoted by $D(x, y, \sigma)$. The DoG is computed as the difference of two consecutive scales, which are separated by constant factor $k$ in a scale space (see Equation (3)).

$$
\begin{aligned}
D(x, y, \sigma) & =(G(x, y, k \sigma)-G(x, y, \sigma)) * I(x, y) \\
& =L(x, y, k \sigma)-L(x, y, \sigma)
\end{aligned}
$$

The scale space is split into octaves. After all the scale-space images have been computed in one octave, the next octave first scale scale-space image is calculated as a sub-sampled version of the last scale-space image in the previous octave.

For computing the local maximums and local minimums in a scale-space three consecutive DoG images in an octave are needed. As illustrated in Figure 6 a pixel in a DoG image, marked by $X$ is compared to its $3 \times 3 \times 3$ neighbourhood in the current and two neighbouring scales. If the pixel is a maximum or a minimum, then the pixel is considered as a candidate keypoint.

The original SIFT implementation ${ }^{4}$ used the following hyper-parameters in the scale-space extrema detection part: initial $\sigma$ was set to 1.6 , the number of octaves was 4 , the number of intervals (scales) in each octave was 5 , and the parameter $k$ was set to $\sqrt{2}$.

\section{2 | Keypoint localization}

In this stage, the candidate keypoints localization is determined and a set of keypoints are filtered by eliminating the parasitic keypoints with low contrast or points on edges.

In Lowe ${ }^{4}$ Taylor series expansion has been used to get a more accurate localization of the candidate keypoint. The expansion of the function $\mathrm{D}(\mathrm{x}, \mathrm{y}, \sigma)$ is computed around the $(x, y)$ candidate keypoint. The precise position of the extremum (DoG position) is then calculated from the derivative of this Taylor expansion.

To filter our parasite keypoints candidate keypoint DoG response is compared to a threshold. If the magnitude of the maximum or minimum of DoG function in the keypoint is smaller than the given threshold (e.g., 0.03 as in reference implementation ${ }^{4}$ ), then the candidate keypoint is rejected.

The DoG response is also strong on the image edges. Candidate keypoints situated on an edge should be removed, as the edges often have aliasing effects and these keypoints are not stable. To discard those candidate keypoints Hessian matrix $H$ is used to determine the principal curvature around the given DoG point.

$$
H=\left[\begin{array}{ll}
D_{x x} & D_{x y} \\
D_{x y} & D_{y y}
\end{array}\right]
$$


Here $D$ are $2^{\text {nd }}$ order partial derivatives. The curvature is computed considering the eigenvalues of the Hessian matrix. Let $\alpha$ and $\beta$ be the two eigenvalues of the matrix. In that case the sum and the product of the eigenvalues are as follows:

$$
\begin{aligned}
\operatorname{Tr}(H) & =D_{x x}+D_{y y}=\alpha+\beta \\
\operatorname{Det}(H) & =D_{x x} D_{y y}-\left(D_{x y}\right)^{2}=\alpha \beta
\end{aligned}
$$

If the determinant of $H$ is less than 0 , then the curvatures have different signs, so the point is a parasite keypoint and has to be discarded.

Supposing that $\alpha$ is the largest magnitude eigenvalue and denoting by $r$ the ratio between the largest magnitude eigenvalue and the lower one, then $\alpha=r \beta$, in that case:

$$
\frac{\operatorname{Tr}(H)^{2}}{\operatorname{Det}(H)}=\frac{(\alpha+\beta)^{2}}{\alpha \beta}=\frac{(r \beta+\beta)^{2}}{r \beta^{2}}=\frac{(r+1)^{2}}{r}
$$

Now we can check the ratio of principal curvatures if it is below some threshold or not:

$$
\frac{\operatorname{Tr}(H)^{2}}{\operatorname{Det}(H)}<\frac{(r+1)^{2}}{r}
$$

If the ratio is larger than the given threshold, the point is a parasitic keypoint on the border and is discarded. In original implementation ${ }^{4} r=10$ was used.

The last two steps concern SIFT descriptor computation. The first step consists in assignment of the orientation to the keypoint to further use it in the rotation-invariant descriptor computation. The last one is the descriptor computation which represents a histogram of orientations of gradients in the vicinity of a detected point, weighted by the magnitude of the gradient. In our solution these two steps are implemented with a reference software on a host computer accordingly to the original algorithm and we do not detail them here.

\section{4 | SIFT IMPLEMENTATION}

In this section we present our hybrid Hardware/Software implementation of SIFT detector and descriptor computation and focus on the parts which have been implemented in FPGA.

At the first step execution time of the SIFT algorithm using the GITW dataset ${ }^{25}$ was measured on the ARM Cortex-A53 CPU of the Xilinx ZCU102 board and on an Intel Xeon E5-2620 server CPU for reference. Average execution time for the SIFT keypoints detection part was 193 and $46 \mathrm{~ms}$ on the ARM Cortex-A53 CPU and the Intel Xeon E5-2620 CPU, respectively. The descriptor computation part can be performed faster. It is executed in 62 and $17 \mathrm{~ms}$ on the CPU architectures. Based on the time required to execute different steps of the algorithm the system was partitioned between the FPGA and the embedded microprocessor. Considering these experimental results the bottleneck of the algorithm is the keypoints detector part; therefore, it is selected for acceleration on the FPGA as it is illustrated in Figure 4.

Figure 4 shows our solution. The first four steps: Gaussian Filtering and DoG computation (GFDG), ScaleSpace Extrema Search (SSE), Non-maximum Supression (NMS), filtering out points on edges, which we call "edge detection" for simplification (ED). These four steps constitute the SIFT point detection part. It is running on the FPGA. The SIFT descriptor generation part is running on the CPU. The CPU and the FPGA SIFT module are communicating using the Advanced Microcontroller Bus Architecture Advanced eXtensible Interface 4 (AMBA AXI4) ${ }^{26}$ protocol.

The development of our FPGA-based SIFT implementation is based on two open source solutions: the OpenCV SIFT function ${ }^{11}$ and the OpenSIFT library. ${ }^{12}$ They were used as a reference code. 


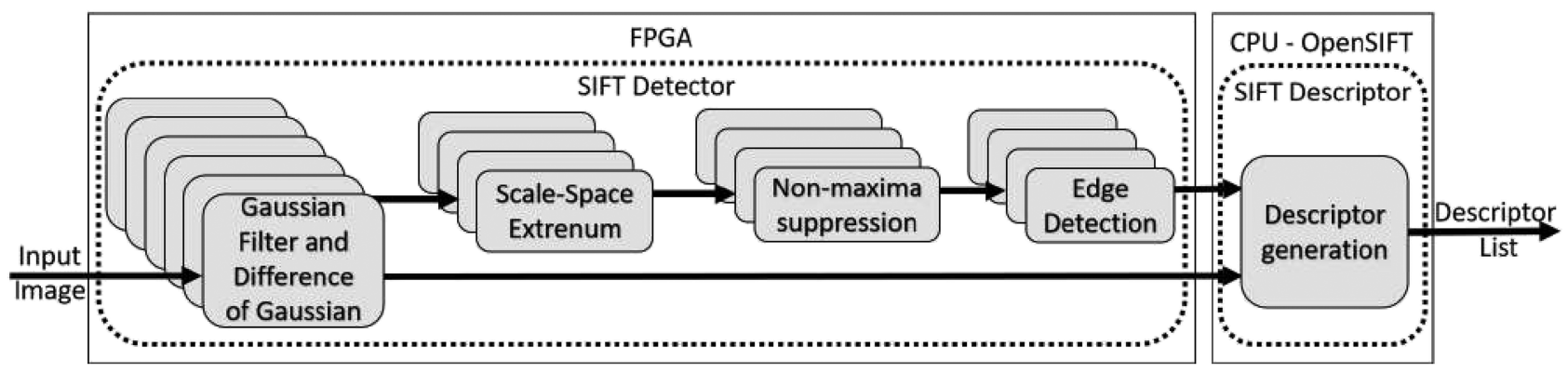

F I G U RE 4 The block diagram of our proposed architecture. The Scale Invariant Feature Transform (SIFT) detector part contains the Gaussian Filtering and Difference of Gaussians computation (GFDG) computation, Scale Space Extrema (SSE), Non-maximum Supression (NMS) and Edge detector modules. The SIFT descriptor part contains the orientation assignment and other descriptor computation steps

\section{1 | GFDG computation module}

This module computes the Gaussian filtered images (see Equation (1)) and the DoG (see Equation (3)) in an octave. Also handling of the border extension (padding) is performed here. It comprises delay arrays which are required for the synchronization of partial results.

The initial scale parameter $\sigma$ in a Gaussain filter (see Equation (2)) is a predefined value and from this the weights of the Gaussian kernel are computed. The Gaussian kernel size is the size of the convolution kernel, of $5 \times 5$ in our case. During the Gaussian computation the method proposed by Shao et al is used. Several Gaussian kernel computations are cascaded in a pipeline and each stage is working on the result of the previous stage. The main input of each pipeline module is the image filtered in the previous stage (or the original image in case of the first stage). For synchronization purposes the results of the previous stages (Gaussian filtered images, DoG images) are also loaded and buffered. The outputs of the module are: the filtered image, the DoG between the image filtered in the previous and the current stage. The buffered results from the previous stages are also sent to the next stage. After the CDFG module all these results will be processed by the SSE module. The results computed by the different stages of the CDFG module can be synchronised utilising this structure.

The border adder/padding adds a frame around the input image. This is necessary for convolution computation at border pixels. The OpenCV ${ }^{11}$ default border padding is called BORDER_REFLECT_101 and performs the padding by a mirror reflection. In that case if the input image is abcdefgh then the output is gfedcb $\|$ abcdefgh $\|$ gfedcba. Therefore, the implementation uses $5 \times 5$ kernel in the Gaussian convolution, two extra rows and columns are added around the image. In our FPGA implementation we also perform the same mirror padding.

The GFDG computation module can convolve an input image with a given Gaussian kernel and substract the result from the input image to compute the DoG image. To utilise the computing power of the FPGA the 2D sliding windows technique is used similarly as in. ${ }^{27}$ In a general case when the size of the window is $N \times N$ and the size of the image is $W \times H$, the last $N-1$ rows from the image must be stored on the FPGA in an $(N-1) \times W$ sized array. The process is illustrated in Figure 5 where the kernel size is $N=3$, and the image width $W=5$ and height $H=6$ for the sake of simplicity.

In a single clock cycle a new pixel data can be written into and an old pixel data can be read from the row memory in parallel with the computation of a new result. This memory arrangement makes feasible pipeline processing of the image.

The input image data of the Gaussian filter is stored in input image window which reads data from the temporary memory as shown in Figure 5. The resulting array can be found below the Gaussian filter. The green squares indicate the data which will be read next time, the red square indicates the being currently processed and written data and the blue squares indicate the currently read data.

\subsection{Scale space extrema search module}

The Scale Space Extrema detection module works in the following way. The current pixel in $3 \times 3 \times 3$ volume (showed in Figure 6) checked that the absolute value of the given pixel is higher than a predefined contrast threshold 
(see Section 6). If the pixel value is greater than the fixed contrast threshold then the next step is to check that the given point is higher or smaller than 0 . If it is larger than 0 and the pixel is a maximum in the given range of $3 \times 3 \times 3$, then the point is a candidate keypoint. If the given pixel is less than 0 than the current pixel is checked whether it is a minimum or not. If it is the minimum then the point is a candidate keypoint. If the given point is neither the maximum nor the minimum, the candidate point is rejected.

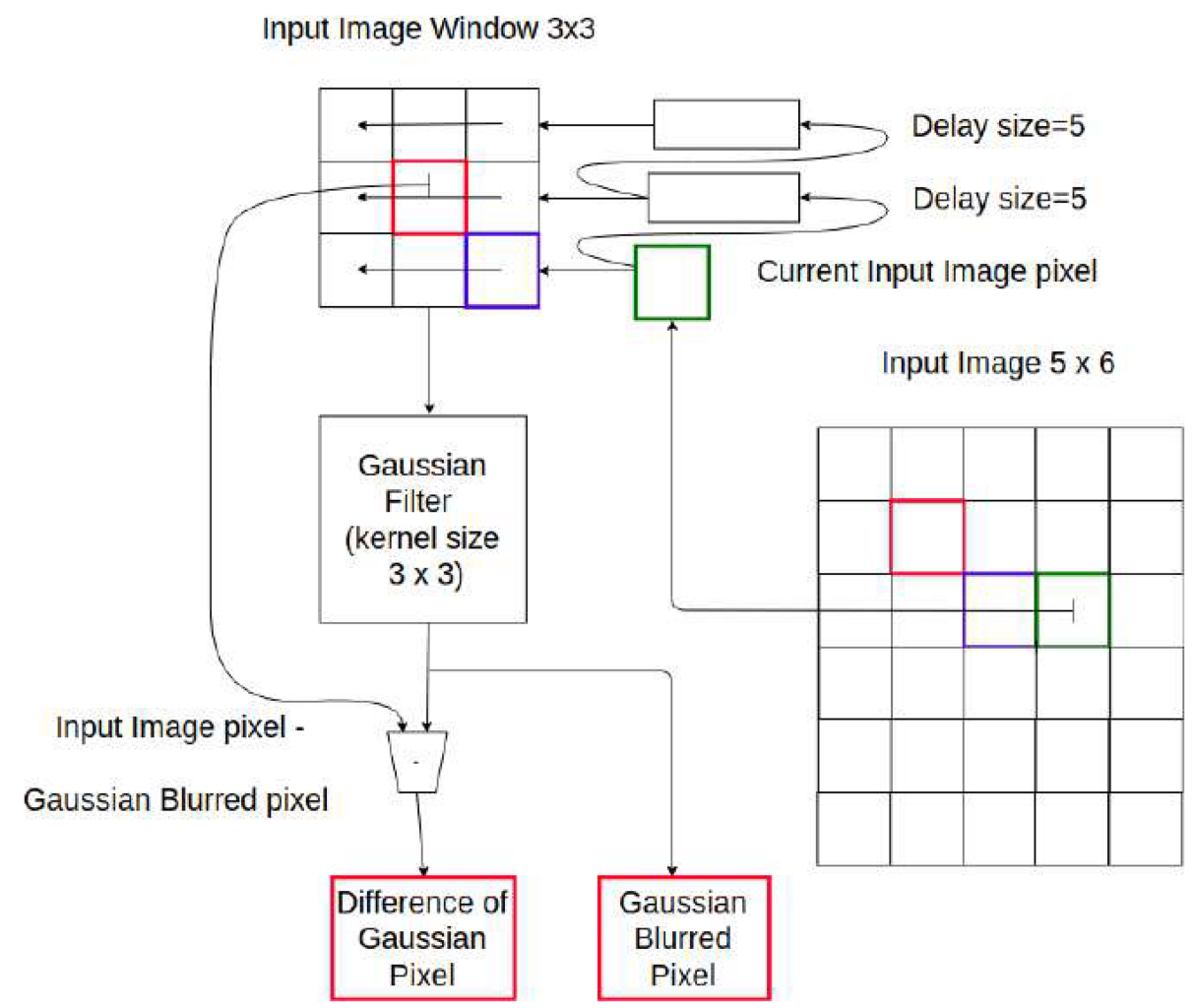

F I G U R E 5 Gaussian Filter and Difference of Gaussian computation module (GFDG). The red rectangle is the current computed pixel. The green rectangle is the current input pixel from the input stream. Neighbours of the currently computed pixel are stored in the input image window. Two lines of the most recently used pixels are stored in the "temporary row delay arrays." Gaussian blurred image and the Difference of Gaussian image are the outputs of the module [Colour figure can be viewed at wileyonlinelibrary.com]

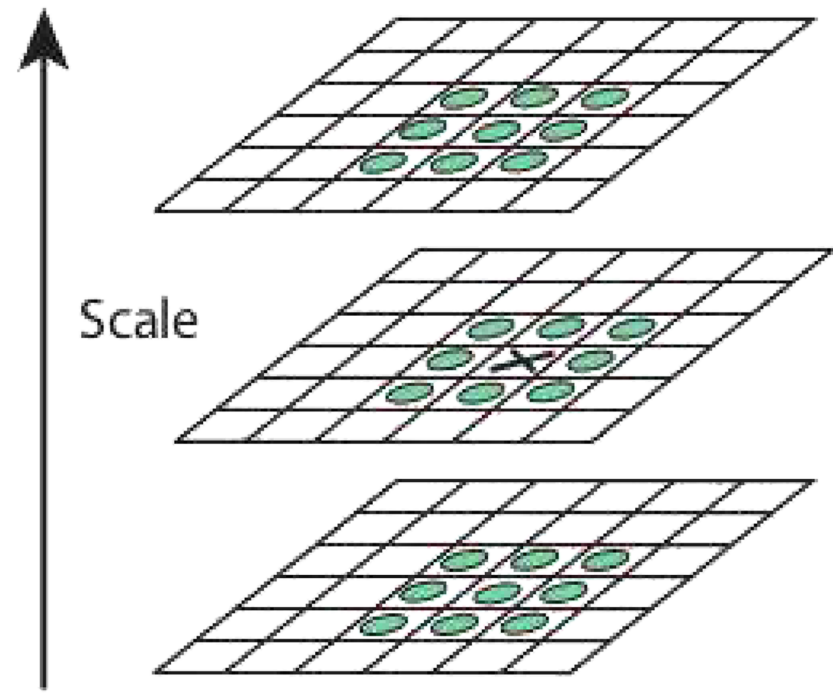

F I G URE 6 Calculate the local extremas in one scale from Lowe paper $^{4}$ [Colour figure can be viewed at wileyonlinelibrary.com] 
Similar 2D windowing technique is used in this module as in the GFDG module (see Figure 5) to efficiently generate the neighbourhood of a pixel. Synchronised results of the preceding three DoG computations are stored in a $2 \times 3 \times W$ row buffer to generate the $3 \times 3 \times 3$ window around each processed pixel. The Gaussian filter computation is replaced by the threshold detection circuit to mark candidate keypoints.

\section{3 | NMS module}

In this subsection the simplification of the SIFT point detection algorithm is presented that is proposed for the FPGA. It consists of the choice of location of a relevant keypoint by a NMS. Instead of computing Taylor expansion (in keypoint localization step) in our FPGA implementation a NMS is proposed. The principle is based on the experimental analysis of detected keypoints with standard settings. It has been shown that many parasite points have been detected despite filtering in the vicinity. This filtering was done at important SIFT points situated on the details of images. Furthermore, for matching of video frames, we do not need sub-pixel accuracy which is proposed by Lowe ${ }^{4}$ when computing Taylor expansion of the DoG and searching for its extrema. NMS can be computed by using absolute value and comparison operations only and no multipliers are required as in the case of Taylor series expansion. This simplification reduces the number of FPGA resources required to implement this part of the algorithm. We can simply express this algorithm as follows.

Lets us consider a given detected point $x_{d p}$ on a discrete scale-image grid and its DoG Response value $D\left(x_{d p}\right)$. Let us consider $\Omega\left(x_{d p}\right)$ a $n \times n$ neighbourhood of $x_{d p}$ in the current octave. We call it NMS kernel. Let us denote with $t h_{D o G}$ a threshold value which suppress low activation value. Let us introduce a function $f: \Omega \rightarrow \mathbb{R}$ :

$$
f\left(x_{d p}\right)=\left\{\begin{array}{cc}
1 & \text { if } \forall x \in \Omega \Rightarrow\left|D\left(x_{d p}\right)\right| \geq|D(x)| \\
0 & \cap\left|D\left(x_{d p}\right)\right|>t h_{\text {DoG }} \\
0 & \text { otherwise }
\end{array}\right.
$$

So the keypoint is rejected if it is not an absolute maximum in magnitude of response in its scale-space neighbourhood at a given scale. Hence, instead of shifting the keypoints by Taylor Expansions, we keep them and remove parasite keypoints with lower absolute response situated too close to the keypoints with stronger responses.

Hence at this step we need two parameters:

- the size $n$ of the neighbourhood $\Omega\left(x_{d p}\right)$ and

- the threshold $t h_{D o G}$ value

In our work we use $n=5$ and the $t h_{D o G}$ is set to 0.01 and different values were also tested as it will be presented in results section.

The module uses 2D sliding window technique, to reduce the number of off-chip memory accesses and to make pipelining possible on the FPGA. The input of the module is as follows: The given DoG image. The outputs of the module is: List of Candidate keypoints.

\section{4 $\quad$ Edge detector module}

This module checks if a candidate keypoint is situated on an edge or not. In the former case it is removed from a list of candidate points. Accordingly, to the SIFT approach Hessian matrix (see Equation (4)) is calculated in the vicinity of the given point. The elements of the Hessian matrix are computed as follows, here we denote by DOG the array containing the DoG values $D(x, y)$ (see Equation (3)) of the given scale and interval:

$$
\begin{aligned}
D_{x x} & =D O G_{[1][2]}+D O G_{[1][0]}-2 \times D O G_{[1][1]} \\
D_{y y} & =D O G_{[2][1]}+D O G_{[0][1]}-2 \times D O G_{[1][1]} \\
D_{x y} & =\frac{D O G_{[2][2]}+D O G_{[2][0]}-D O G_{[0][2]}+D O G_{[0][0]}}{4}
\end{aligned}
$$


To store the required DoG elements around the candidate keypoint a 2D sliding window temporary memory is used in the module. The determinant of Hessian matrix calculated and if the determinant greater than 0 , and the Hessian matrix trace sum squared is less than a predefined $\epsilon$ value the candidate keypoint still remains a candidate keypoint accordingly to the SIFT algorithm, see Section 3. The $\epsilon$ value is calculated from the curvature threshold.

$$
\epsilon=\frac{(\text { curvature threshold }+1.0)^{2}}{\text { curvature threshold }} \times \operatorname{det}(H)
$$

In other case the candidate keypoint is removed from the keypoint list, as belonging to a border and not to a corner.

Inputs of this module are: DoG image at the given candidate keypoint, list of the candidate keypoints, the previous results. Outputs of this module are as follows: filtered list of the candidate keypoints.

\section{5 | EXPERIMENTS AND RESULTS}

In this paper the implementations is compared in terms of precision of detection of keypoints, processing speed and dissipation power. The baseline for validation of different modules developed was the OpenSIFT implementation. ${ }^{12}$ The experiments were conducted on the "Grasping-in-the-wild (GITW) dataset ${ }^{25}$ " freely available for research at NAKALA CNRS server. ${ }^{1}$ It is describe it in the following.

\section{1 | Dataset}

The dataset GITW has been recorded for development of vision-based hybrid neuro-prostheses of new generation. ${ }^{1}$ The dataset contains egocentric videos recorded by a camera worn on a shoulder of a person and on the eye-tracker glasses. The data set is recorded in a natural cluttered everyday life environment, that is, kitchens. The goal of SIFT point detection is matching of views by homography computation. In the visual scenario subjects are grasping objects of everyday life like a mug or a bowl. The duration of videos is about $10 \mathrm{~s}$, the recording devices are eye-tracker with camera Tobii Glasses 2 and shoulder-mounted GoPro cameras. The resolution of the glass videos are HD (1280 pixels $\times 720$ pixels), and the frame rate of the videos is $25 \mathrm{fps}$. The GoPro recorded videos resolution is Full HD (1920 pixels $\times 1080$ pixels), and $60 \mathrm{fps}$ frame rate. The dataset contains 404 egocentric videos. It has 16 different categories of the recorded objects: Bowl, Plate, Wash Liquid, Vinegar Bottle, Milk Bottle, Oil Bottle, Glass, Lid, Saucepan, Frying Pan, Mug.

The experiments were conducted on a subset of the whole dataset. The Bowl subdataset was chosen for this project. This subdataset contains 18 different "cases." Each case contains an egocentric video and a bounding_box.txt file. The bounding_box.txt file contains the coordinates of a bounding box of the object of interest in each frame. All videos are recorded by different subjects in five different places. The overview of the "Bowl" subdatset is presented in the Table 1 in terms of duration, number of frames and number of bounding boxes for the Bowl object in videos.

Figure 7 depicts some example videos from the Bowl subset. In Place1 videos, each subject grasps a red bowl. These videos have a moderate complexity as the videos does not contain a large amount of other objects than bowl and the background has a different colour from the bowl. In Place4 videos contain a blue bowl as the object of interest. The environment is cluttered with objects including the target bowl. A cup and a milk bottle have the same colour as the bowl. In this scenario the bowl is dark it has a similar colour to the background. The scene contains occlusions. Subject in Place5 videos grasps a transparent glass bowl. There are some other objects and the bowl is behind a transparent glass. Due to the fact that the object of interest is transparent the object and the background are highly similar. In Place6 videos show a subject grasping a ceramic beige bowl. The video includes other objects too however, the bowl is in front of the subject so there is no occlusion. The bowl has different colour than the other objects and the background. The last video sequence is recorded in Place7. A subject grasps a transparent glass bowl. There is no other objects in the camera field-of-view. The bowl is really similar to the background. As the target application is frame matching with SIFT points, then obviously the more SIFT points are situated on the object of interest better will be robustness of Homography computation. In case of occlusions, transparencies or similarity with the background the SIFT points on objects only will not be sufficient. This is why the detection of SIFT points is proposed not only in the bounding boxes of

${ }^{1}$ https://www.labri.fr/projet/AIV/graspinginthewild.php 
T A B L E 1 Bowl subdataset overview

\begin{tabular}{|c|c|c|c|c|}
\hline Number & Foldername & Duration (s) & Number of frames & Bounding Box objects \\
\hline 2 & BowlPlace1Subject2 & 7.12 & 179 & 165 \\
\hline 3 & BowlPlace1Subject3 & 5.48 & 138 & 27 \\
\hline 4 & BowlPlace1Subject4 & 5.64 & 142 & 59 \\
\hline 7 & BowlPlace4Subject3 & 12.36 & 310 & 151 \\
\hline 8 & BowlPlace4Subject4 & 7.84 & 197 & 107 \\
\hline 9 & BowlPlace5Subject1 & 5.76 & 145 & 98 \\
\hline 10 & BowlPlace5Subject2 & 9 & 226 & 124 \\
\hline 14 & BowlPlace6Subject3 & 12.08 & 303 & 64 \\
\hline 15 & BowlPlace6Subject4 & 6.72 & 169 & 63 \\
\hline 16 & BowlPlace7Subject1 & 11.44 & 287 & 89 \\
\hline 17 & BowlPlace7Subject2 & 9.48 & 238 & 58 \\
\hline 18 & BowlPlace7Subject3 & 4.88 & 123 & 38 \\
\hline Total & N/A & 153.68 & 3860 & 1740 \\
\hline
\end{tabular}
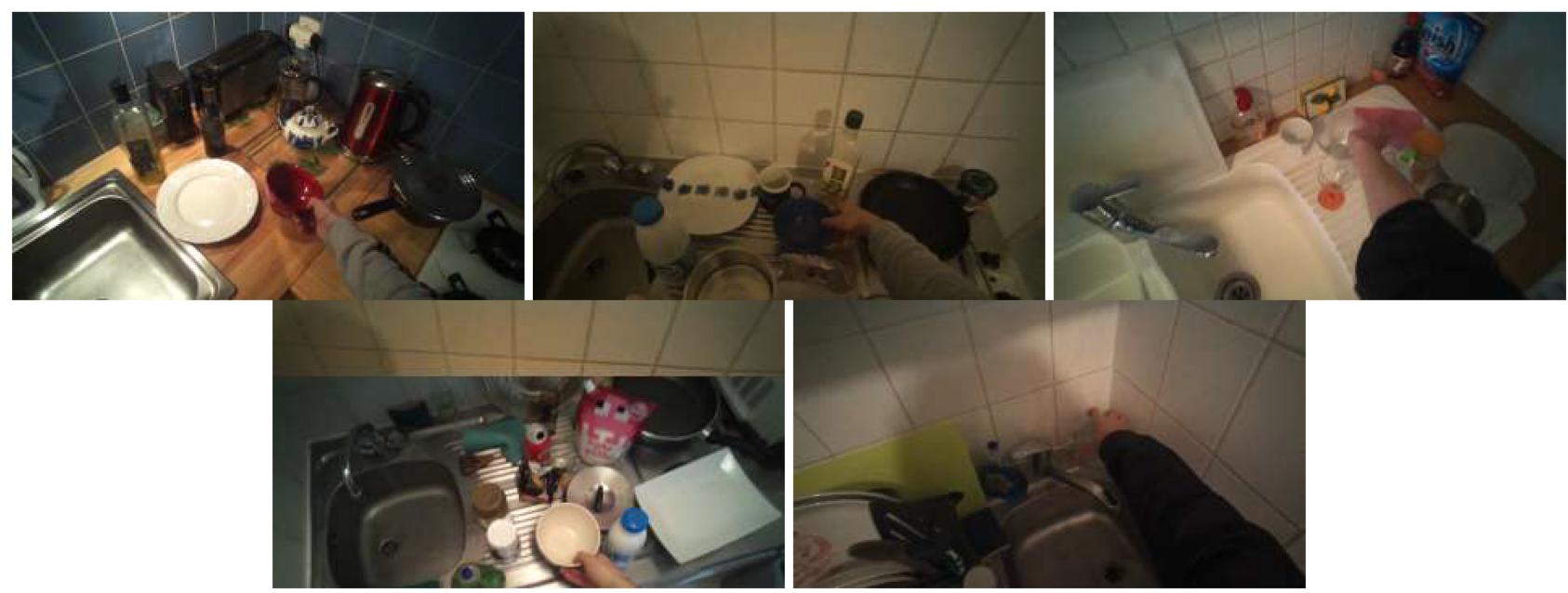

F I G U RE 7 Examples of the Bowl subset video scenes. Top left is BowlPlace1Subject2, the center is BowlPlace4Subject2, the top right is BowlPlace5Subject2 the bottom left is BowlPlace6Subject2, and the bottom right is BowlPlace7Subject2 scene [Colour figure can be viewed at wileyonlinelibrary.com]

objects but in a slightly extended area around bounding box. This extension should not be very large due to the depth variations in the scene the homography model will not be sufficient. Thus, an augmented bounding box was used with an area of $480 \mathrm{px} \times 480 \mathrm{px}$ size.

During the experiments the default parameter list can be found in Table 2. The first seven parameters are used both by the OpenSIFT ${ }^{12}$ and our FPGA implementations. The FPGA only parameters are used by our FPGA implementation. 


\begin{tabular}{|c|c|}
\hline Name & Value \\
\hline image size & $480 \mathrm{px} \times 480 \mathrm{px}$ \\
\hline octave & 3 \\
\hline scale & 6 \\
\hline Gaussian kernel size & $5 \times 5$ \\
\hline initial $\sigma$ & 1.6 \\
\hline contrast threshold & 0.04 \\
\hline curvature threshold & 10 \\
\hline FPGA only parameters & \\
\hline NMS kernel size & $5 \times 5$ \\
\hline th $h_{\text {DoG }}$ & 0.01 \\
\hline
\end{tabular}

TABLE 2 The default parameter list in experiments

\section{2 | GFDG module validation}

To validate the GFDG module we focus on the comparison of the Gaussian filtering implemented in FPGA with regard to the reference software. The GFDG module was compared to the OpenCV 2.4.13.7 cvSmooth function. This function is convolving an image with a Gaussian kernel and the output of the function is a Gaussianconvolved image. On the FPGA board OpenCV 4 has been used and the cvSmooth function renamed to GaussianBlur. This result and the module result are compared to each other and if the absolute difference between the two results is less than a predefined threshold $\epsilon_{1}$ the module is validated. Hence for each frame $I_{m}, m=1, \ldots, M$ in our dataset the first computation is the maximal value $M D_{m}$ of the absolute pixel values difference between the two results $d I_{m}$ :

$$
M D_{m}=\max _{(x, y)}\left(\left|O C V_{G}\left(I_{m}(x, y)\right)-F P G A_{G}\left(I_{m}(x, y)\right)\right|\right)
$$

Here $O C V_{G}$ is the OpenCV cvSmooth function, $F P G A_{G}$ is the GFDG module. Then the mean maximal absolute difference on the whole dataset $M M D$ is computed:

$$
M M D=\frac{1}{M} \sum_{m=1}^{M} M D_{m}
$$

with $M$ is the number of video frames in the whole dataset.

In the simulation of the Vivado HLS 2018.328 shows the MMD between the GFDG module and the OpenCV 2.4.13.7 cvSmooth function are smaller than $\epsilon_{1}=1 e-5$ in the whole bowl dataset.

\section{3 | Comparison of the CPU implementations with FPGA implementations}

To illustrate the behaviour of our simplified SIFT detector implementation in FPGA and in comparison with OpenSIFT $^{12}$ we present the number of detected points on each frame of one sequence from our dataset in Figure 8 for both detectors. As we expected the FPGA implementation detects more keypoints.

The video sequence in this experiment is the BowlPlace6Subject2 glass video. During this experiment the default parameters (Table 2) have been used. In Figure 9 an example is given which show the detection for one frame of a video for the seek of visual comparison both by OpenSIFT ${ }^{12}$ and the FPGA implementations. One can see that the keypoint sets are quite similar. The difference is coming from the fact that ${ }^{12}$ uses Taylor expansion in the keypoint localization step, and the FPGA implementation uses the Non-Maximum Suppression only, see Section 4. 


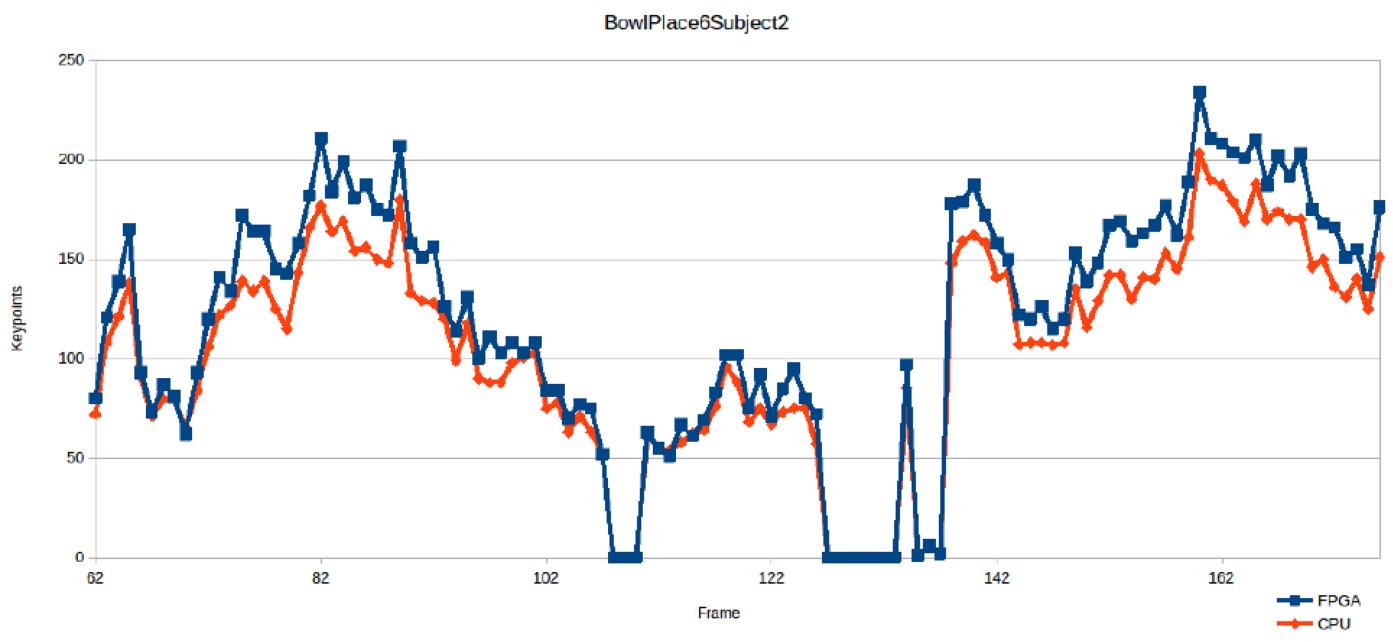

FI I URE 8 Comparison between the ${ }^{12}$ and the FPGA implementation detected keypoints. $Y$ axis is the number of the extracted keypoints, $X$ axis is the frame number. FPGA-detected keypoints are in blue, CPU-detected keypoints are in red [Colour figure can be viewed at wileyonlinelibrary.com]
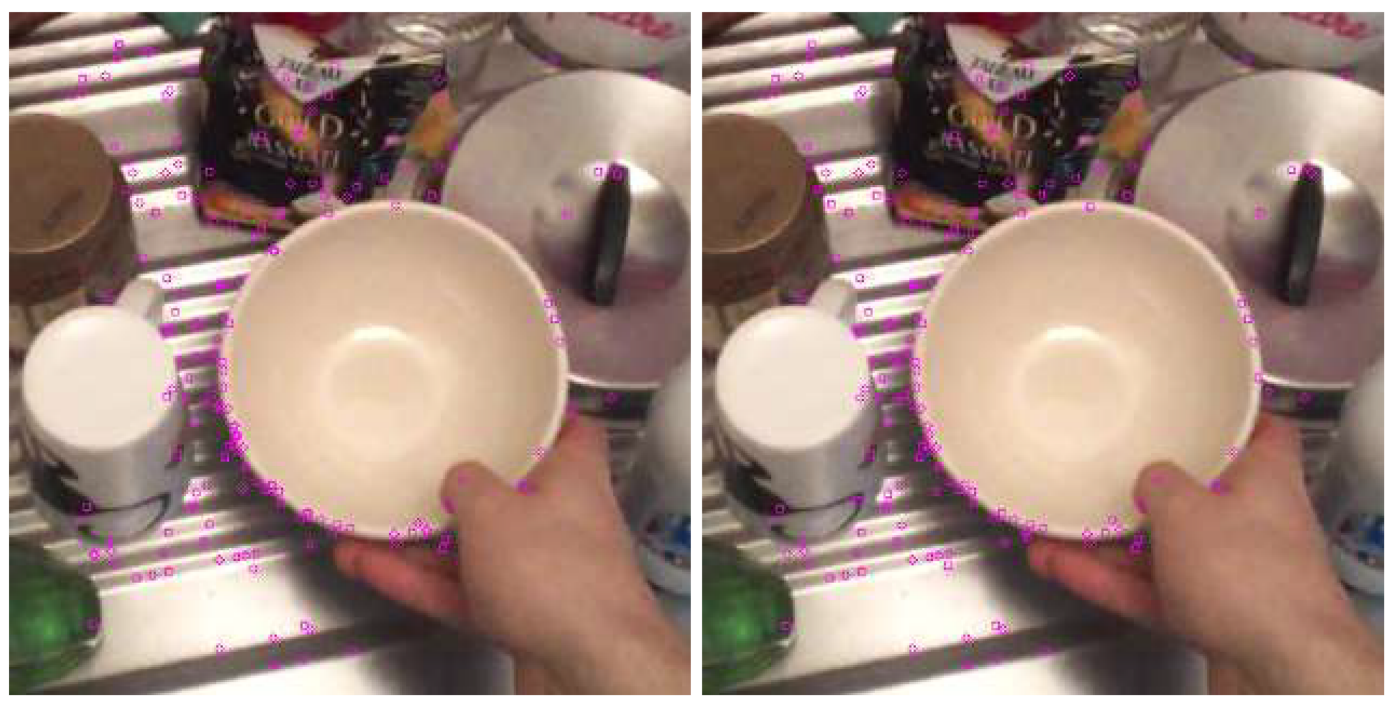

F I G U RE 9 Visual comparison of detected sets of keypoints. On the left: OpenSIFT ${ }^{12}$ extracted keypoints. On the right: the FPGA result [Colour figure can be viewed at wileyonlinelibrary.com]

\subsection{The FPGA keypoint detection module assessment}

Here we compare the overall results of detection of SIFT keypoints by our FPGA solution with detection by the reference software OpenSIFT. ${ }^{12}$ We consider that two keypoints (FPGA-detected and OpenSIFT-detected) coincide if the Manhattan distance between them is less than a predefined threshold $\epsilon_{2}$. As a threshold value we have taken 2 . Hence,

\footnotetext{
True Positive : $x_{C P U} \exists x_{F P G A}:\left\|x_{C P U}-x_{F P G A}\right\|<\epsilon_{2}$

False negative : $x_{C P U} \cap\left(x_{F P G A}=\emptyset\right)$

False positive : $x_{F P G A} \cap\left(x_{C P U}=\emptyset\right)$

True negative : $\emptyset$ CPU and $\emptyset$ FPGA
}

where $x_{C P U}$ is a keypoint in ${ }^{12}$ and $x_{F P G A}$ is a keypoint in the FPGA implementation. True positive (TP) is the case when the distance between the $x_{C P U}$ and $x_{F P G A}$ is less than a predefined threshold $\epsilon_{2}$. False negative (FN) is when no $x_{F P G A}$ 
points in a radius $\epsilon_{2}$ circle in a center $x_{C P U}$. False postive (FP) is when no $x_{C P U}$ points in a radius $\epsilon_{2}$ circle in a center $x_{F P G A}$. True negative (TN) corresponds to the case when there is no keypoint in both of the implementations.

Precision (P) and recall (R) are calculated accordingly.

$$
\begin{aligned}
& P=\frac{T P}{T P+F P} \\
& R=\frac{T P}{T P+F N}
\end{aligned}
$$

Table 3 illustrates the results when default parameters have been used, see Table 2 . The first column contains the name of the video sequences. The second column depicts the average number of OpenSIFT ${ }^{12}$-extracted keypoints in the frames of a video sequence and the standard deviation. The average per/frame number of our FPGA-extracted keypoints and the standard deviation are given in the first column. The final two columns are the average precision and the average recall. It can be seen that the numbers of recall are quite high - up to 0.97 while precision is lower from 0.77 up to 0.91 . This confirms the general trend that FPGA based implementation with a simplified algorithm increases the number of supplementary detections.

Different parameters have been tested too. The $t h_{D o G}$ has been set to $0,0.008,0.009,0.01,0.011,0.012$. In those cases the recall and the precision were lower then in the experiment with 0.01 value. When the initial scale parameter $\sigma$ was set to 0.45 the average number of extracted keypoints was higher (OpenSIFT ${ }^{12} 167.73$, in our FPGA implementation 102.60). The distance between the keypoints are small, and the NMS removed them. That is why the OpenSIFT ${ }^{12}$ extracted more keypoints than our FPGA implementation in that case. The average recall was 0.58 , which is lower when the initial $\sigma$ was set to 1.6. The average precision was 0.88 , which is the same as when initial $\sigma$ is set to 1.6.

Now we trace the behaviour of our FPGA implementation in different octaves. In case when the initial $\sigma$ was set to 1.6 the average number of extracted keypoints in the $1^{\text {st }}$ octave was 15.93 with OpenSIFT ${ }^{12}$ and 18.83 with our FPGA implementation on the whole bowl subset.

For the second octave, OpenSIFT ${ }^{12}$ has extracted an average number of 11.72 keypoints and our FPGA implementation 12.28 respectively on the whole bowl subset as well. There are some cases when in the second octave more keypoints have been found than in the first octave like BowlPlace1Subject2 and BowlPlace6Subject1. However, the

\begin{tabular}{|lccll}
\hline Name & CPU & FPGA & P & R \\
\hline BP1S1 & $37.08 \pm 25.42$ & $41.21 \pm 27.51$ & $0.79 \pm 0.24$ & $0.93 \pm 0.11$ \\
\hline BP1S2 & $41.66 \pm 32.40$ & $46.63 \pm 34.39$ & $0.77 \pm 0.24$ & $0.92 \pm 0.12$ \\
\hline BP1S3 & $29.02 \pm 16.96$ & $32.62 \pm 18.03$ & $0.81 \pm 0.21$ & $0.92 \pm 0.15$ \\
\hline BP1S4 & $48.61 \pm 30.85$ & $53.67 \pm 32.95$ & $0.83 \pm 0.17$ & $0.93 \pm 0.08$ \\
\hline BP4S1 & $13.99 \pm 16.67$ & $15.49 \pm 17.62$ & $0.85 \pm 0.25$ & $0.9 \pm 0.21$ \\
\hline BP4S2 & $11.21 \pm 7.80$ & $11.94 \pm 8.25$ & $0.86 \pm 0.21$ & $0.93 \pm 0.16$ \\
\hline BP4S3 & $38.54 \pm 24.42$ & $42.39 \pm 26.54$ & $0.86 \pm 0.16$ & $0.94 \pm 0.08$ \\
\hline BP4S4 & $3.92 \pm 8.13$ & $4.25 \pm 9.33$ & $0.89 \pm 0.25$ & $0.92 \pm 0.23$ \\
\hline BP5S1 & $17.43 \pm 18.12$ & $19.09 \pm 18.92$ & $0.83 \pm 0.24$ & $0.93 \pm 0.14$ \\
\hline BP5S2 & $9.27 \pm 11.01$ & $10.29 \pm 11.93$ & $0.83 \pm 0.24$ & $0.93 \pm 0.15$ \\
\hline BP5S3 & $20.59 \pm 15.46$ & $22.54 \pm 16.91$ & $0.86 \pm 0.16$ & $0.94 \pm 0.11$ \\
\hline BP6S1 & $73.51 \pm 45.45$ & $83.12 \pm 51.17$ & $0.85 \pm 0.12$ & $0.94 \pm 0.06$ \\
\hline BP6S2 & $105.92 \pm 56.04$ & $120.64 \pm 65.22$ & $0.85 \pm 0.07$ & $0.93 \pm 0.06$ \\
\hline BP6S3 & $77.79 \pm 48.74$ & $88.50 \pm 55.44$ & $0.84 \pm 0.16$ & $0.95 \pm 0.06$ \\
\hline BP6S4 & $22.70 \pm 46.15$ & $26.05 \pm 52.62$ & $0.77 \pm 0.23$ & $0.92 \pm 0.11$ \\
\hline BP7S1 & $2.48 \pm 4.22$ & $2.61 \pm 4.45$ & $0.91 \pm 0.22$ & $0.91 \pm 0.22$ \\
\hline BP7S2 & $4.38 \pm 7.14$ & $5.00 \pm 8.04$ & $0.84 \pm 0.27$ & $0.95 \pm 0.14$ \\
\hline BP7S3 & $4.55 \pm 8.22$ & $5.10 \pm 8.99$ & $0.84 \pm 0.29$ & $0.97 \pm 0.09$ \\
\hline
\end{tabular}

TABLE 3 Comparison of the number of keypoints in case of OpenSIFT (CPU) ${ }^{12}$ and our FPGA implementations. $\mathrm{P}$ is the Precision and $\mathrm{R}$ is the Recall 
extracted keypoints number decreased in the $3^{\text {rd }}$ octave (OpenSIFT12 3.61 keypoints in average and our FPGA implementation 3.96 keypoints in average respectively) compared to the $1^{\text {st }}$ and $2^{\text {nd }}$ octaves. Thus, the behaviour of our SIFT detector implemented in FPGA is generally the same: it gives more points than reference OpenSIFT implementation in different octaves.

These results showed that our FPGA and the full-software OpenSIFT ${ }^{12}$ keypoints are almost equivalent. In that case equivalent means that the keypoints in the reference images are really close to each other and situated at a distance less than the predefined threshold of 2 pixels.

\section{5 | Used resources}

The resource usage of the complete system is presented in Table 4. The implementation is based on the Vivado $2018.3^{28}$ software, which can report the hardware requirements and clock frequency of the circuit. Here the default parameters presented in Table 2 have been used. The results show that around 50\% of the resources have been used on the Xilinx ZCU 102 FPGA board. Therefore there are enough resources to implement other steps of the prosthetic arm controlling algorithm.

The system is implemented in Vivado HLS as a single IP block. The Vivado toolchain does not hold the details of this block's exact inner structure during the implementation step. Therefore, the area requirements of the submodules are summarised using the Vivado HLS area report as shown in Table 5. The complete system is build up using six GFDG modules, four SSE modules, four NMS modules and four ED modules. The most resource-consuming part is the GFDG computation module where the majority of the BRAM and DSP slices are used. The area requirements of the SSE and NMS units are dominated by the logic resources (FFs and LUTs).

\section{6 | Discussion}

In this section we compare our method with other platforms and previous solutions.

\subsection{1 | Comparison of FPGA implementation with other platforms}

Table 6 shows how many frames can be processed on the given hardware per second. Our FPGA solution is reported in the column 1 (Xilinx UltraScale+ ZCU102). The result shows FPGAs, Intel Xeon E5-2620 server CPU, GPU and the

TA B L E 4 One octave resource usages on Xilinx ZCU102 FPGA Board from Vivado 2018.328 when the default parameters in Table 2 has been used

\begin{tabular}{|lccc|}
\hline Resource \# & Full System & SIFT computation module & Available \\
\hline LUT & 143,667 & 117,620 & 274,080 \\
\hline FF & 189,944 & 157,946 & 548,160 \\
\hline BRAM & 461.5 & 461.5 & 912 \\
\hline DSP & 938 & 938 & 2,520 \\
\hline
\end{tabular}

TA B LE 5 Resource usage estimation of the main modules of the system based on the Vivado HLS report

\begin{tabular}{|c|c|c|c|c|c|}
\hline $\begin{array}{l}\text { Resources } \\
\#\end{array}$ & $\begin{array}{l}\text { Gauss filter and Difference of } \\
\text { Gaussian (GFDG) }\end{array}$ & $\begin{array}{l}\text { Scale Space } \\
\text { Extrema (SSE) }\end{array}$ & $\begin{array}{l}\text { Non-Maximum } \\
\text { Suppression (NMS) }\end{array}$ & $\begin{array}{l}\text { Edge Detection } \\
\text { (ED) }\end{array}$ & Total \\
\hline $\mathrm{FF}$ & 17810 & 5569 & 9314 & 6630 & 219243 \\
\hline DSPs & 127 & 0 & 0 & 44 & 938 \\
\hline LUT & 17572 & 7255 & 19026 & 6160 & 276366 \\
\hline
\end{tabular}


CMOS Vision Sensor can process more than 70 frames per second. Our FPGA implementation used the default parameters in Table 2. The Intel Xeon E5-2620 server CPU and the ARM Cortex-A53 were measured with the OpenSIFT ${ }^{12}$ SIFT implementation with the same parameters as our FPGA implementation. The analog solution (CMOS Vision Sensor) computed just the first step of the SIFT algorithm the Gaussian pyramid calculation. However, energy consumption of the Intel Xeon E5-2620 server CPU and the GPU are higher than the FPGAs. The CMOS Vision Sensor solution only handles $176 \times 120$ pixels images which is much smaller than the FPGAs.

The power dissipation of the proposed SIFT module is relatively low, just 5.6 W. The CMOS Vision Sensor has the lowest energy consumption $70 \mathrm{~mW}$, however, the input resolution $(176 \mathrm{px} \times 120 \mathrm{px})$ and the numbers of pixels being processed (2.6 million px) are lower than in the FPGAs.

Comparison of the power consumption shows that the Intel Xeon E5-2620 server CPU, and that the GPU, are using too much energy for a wearable device. In contrast, the power consumption of the FPGA, the analog CMOS Vision Sensor, and the ARM Cortex-A53 CPU, are sufficiently low for our application. However, the processing speed of the ARM Cortex-A53 mobile CPU is too low and does not fulfil our demands for real-time processing. The Intel Xeon E5-2620 CPU, FPGA, GPU, and CMOS Vision sensor are capable of real-time processing. The analog solutions can only process small input images, whereas the FPGA, GPU, and both of the CPUs can handle sufficiently high image resolution which is higher than Full HD. The FPGA solution is equivalent to the full software implementation OpenSIFT ${ }^{12}$ in terms of proximity and number of detected keypoints.

The disadvantage of the FPGA solution is that the input image size is small $480 \mathrm{px} \times 480 \mathrm{px}$, due to the limited $32.1 \mathrm{Mb}$ internal memory of the Xilinx UltraScale+ ZCU102. The CPUs and the GPU's solutions can process higherresolution input. Nevertheless, for controlling the robotic arm this resolution is sufficient.

Comparison of the power consumption, processing speed and input image size for the different architectures shows that the FPGA is the best choice for creating a wearable device as Table 6 shows.

The latency of the system is 238390 clock cycles for the input image size of $480 \mathrm{px} \times 480 \mathrm{px}$ this is based on the Vivado HLS 2018.3 report. ${ }^{28}$ The current implementation is running on 200-MHz clock frequency according to the post routing timing analysis in Vivado 2018.3,28 so it can process 135 full $480 \mathrm{px} \times 480 \mathrm{px}$ images per second, which is higher than the current input video frame rate. Therefore, a real-time processing is achievable.

\subsection{2 | Comparison of the different FPGA implementations}

In this part we compared our implementation to the state-of-the-art FPGA solutions. As Table 7 indicated we used a different FPGA board (Xilinx UltraScale+ ZCU102) from that used in previous studies. Doménech-Asensi et al. ${ }^{15}$ and Shao et al. ${ }^{20}$ use Xilinx Virtex-5. Rubio-Ibáñez et al. ${ }^{16}$ implementation run on Zedboard, and Vourvoulakis et al. ${ }^{17,18}$ developed their solution on Intel DE2i-150. Chang et al.19 developed their solution for a Xilinx Virtex II Pro. Our solution can process a $480 \mathrm{px} \times 480 \mathrm{px}$ resolution image which has a higher resolution compared to the Chang et al. ${ }^{19}$ and Shao et al, ${ }^{20}$ but Doménech-Asensi et al. ${ }^{15}$ and Vourvoulakis et al. ${ }^{17,18}$ implementations can process images with higher resolution of $640 \mathrm{px} \times 480 \mathrm{px}$. Our proposed architecture can compute 135 frames per second, which is higher than both Doménech-Asensi et al. ${ }^{15}$ and Vourvoulakis et al. ${ }^{17,18}$ and Shao et al. ${ }^{20}$ In comparison, Chang et al. ${ }^{19}$ solution can process 900 frames per second; however, it uses lower image resolution. Our implementation is a software/hardware

TA B LE 6 Comparison of SIFT keypoint extraction in different platforms per second

\begin{tabular}{|cccccc}
\hline & $\begin{array}{l}\text { Xilinx UltraScale+ ZCU102 } \\
\text { Our solution }\end{array}$ & $\begin{array}{l}\text { Intel Xeon } \\
\text { E5-2620 }\end{array}$ & $\begin{array}{l}\text { ARM } \\
\text { Cortex-A53 }\end{array}$ & $\begin{array}{l}\text { CMOS Vision } \\
\text { Sensor }\end{array}$ & $\begin{array}{l}\text { NVIDIA GeForce } \\
\text { GTX 580 }\end{array}$ \\
\hline $\begin{array}{c}\text { implemented } \\
\text { algorithm }\end{array}$ & OpenSIFT (FPGA version) & OpenSIFT & OpenSIFT & & $\begin{array}{l}\text { Gaussian } \\
\text { Pyramid }^{21}\end{array}$ \\
resolution (px) & $480 \times 480$ & $480 \times 480$ & $480 \times 480$ & $176 \times 120$ & $1280 \times 960$ \\
\hline frames / second & 135 & 21 & 5 & 125 & 78.74 \\
\hline dissipated power & $5.6 \mathrm{~W}$ & $80 \mathrm{~W}$ & $2.5 \mathrm{~W}$ & $70 \mathrm{~mW}$ & $244 \mathrm{~W}$ \\
\hline
\end{tabular}

Note: The Intel Xeon E5-2620 and ARM Cortex-A53 are used the OpenSIFT12 and the Xilinx UltraScale+ ZCU102 are used our FPGA implementation. All three platform which used OpenSIFT are used with the default parameters (Table 2). On the CMOS Vision Sensor only the time for Gaussian Pyramid calculation is reported. 
TAB L E 7 Comparision of our work with different FPGA implementations

\begin{tabular}{|c|c|c|c|c|c|c|}
\hline & $\begin{array}{l}\text { Our } \\
\text { implementation }\end{array}$ & $\begin{array}{l}\text { Doménech- } \\
\text { Asensi }^{15}\end{array}$ & $\begin{array}{l}\text { Rubio- } \\
\text { Ibáñez }^{16}\end{array}$ & Vourvoulakis $^{17,18}$ & Chang $^{19}$ & Shao $^{20}$ \\
\hline board & ZCU102 & Xilinx Virtex-5 & ZedBoard & Intel DE2i-150 & $\begin{array}{c}\text { Xilinx Virtex II } \\
\text { Pro }\end{array}$ & $\begin{array}{l}\text { Xilinx } \\
\text { Virtex-5 }\end{array}$ \\
\hline resolution (px) & $480 \times 480$ & $640 \times 480$ & - & $640 \times 480$ & $320 \times 240$ & $292 \times 520$ \\
\hline frames/second & 135 & 99 & - & 81 & 900 & 38 \\
\hline $\begin{array}{l}\text { number } \\
\text { representation }\end{array}$ & float & fixed-point & fixed-point & fixed-point & fixed-point & fixed-poin \\
\hline
\end{tabular}

solution similarly to Rubio-Ibáñez et al, ${ }^{16}$ and Chang et al. ${ }^{19}$ and Shao et al. ${ }^{20}$ Other papers such as Doménech-Asensi et al. ${ }^{15}$ and Vourvoulakis et al. ${ }^{17,18}$ use solutions that are a hardware but simplified implementation of the SIFT, which impacts the algorithm accuracy. Our solution is using a float number representation instead of the fixed-point representation. This causes a higher resource demand, but it also provides better accuracy.

\section{6 | CONCLUSION}

Hence in this paper a design and implementation of SIFT detector on FPGA was presented.

The results show that a wearable device can be developed by using FPGA with 135 images per second processing rate, when the input image resolution is of $480 \mathrm{px} \times 480 \mathrm{px}$ and it is running on Xilinx ZCU102 FPGA board.

In our FPGA implementation a simplified keypoint localization method is proposed. Instead of computing Taylor expansion, keypoints too close to each other are filtered using NMS.

Our FPGA implementation and the reference OpenSIFT ${ }^{12}$ software implementation were compared in different ways. The experiments with real examples demonstrated that our FPGA SIFT implementation gives equivalent output to the reference software detector. Indeed, tests on real-world video in cluttered environments yielded the average precision up to 0.84 and the average recall of 0.97 in SIFT point detection when the initial Gaussian scale parameter $\sigma$ was set to 1.6. With a different parameter we got close results, however the recall was lower. Different threshold values of $t h_{D o G}$ parameter in our simplified implementation were tested and we got the best result with 0.01 .

The test system was implemented on Xilinx ZCU102 FPGA board and uses around 50\% of the FPGA resources.

The power consumption of the FPGA is 5.6W which in comparison to high-end CPU is almost 15 times less. It is low enough to use it in a wearable device as for the control of a robotic arm. The FPGA board is a better option to accelerate the algorithms in this project compared to CPUs, GPU, and the analog CMOS Vision sensor. Our measurements (see in Table 6) proved that the FPGA can process the images at the same or at a higher speed as the CPUs, GPUs, and the analog CMOS Vision sensor. Furthermore, the FPGA has a lower energy consumption than the Intel Xeon E5-2620 server $\mathrm{CPU}$, and the GPUs. FPGA based solutions also support higher image resolution compared to CMOS Vision Sensor ${ }^{21,22}$ implementation. and the size of the processed image can be modified similarly to the CPU implementations. ${ }^{11,12}$

Taking into account the results of analysis of our FPGA implementation and its comparison with existing solutions it was concluded that the developed implementation is competitive in terms of keypoints detection precision, and optimal in terms of power consumption, processing speed and frame size. It is thus suitable for the control of a portable mobile robotic arm.

\section{ACKNOWLEDGMENTS}

The support of the Széchenyi 2020 Program, of the Human Resource Development Operational Program, of the Program of Integrated Territorial Investments in Central-Hungary (project numbers: EFOP-3.6.2-16-2017-00013 AND 3.6.3-vekop-16-2017-00002) and of the European Structural and Investment funds, CNRS and Balaton PHC are gratefully acknowledged.

\section{DATA AVAILABILITY STATEMENT}

The data that support the findings of this study are openly available in Grasping In The Wild at https://www.labri.fr/ projet/AIV/graspinginthewild.php. 


\section{ORCID}

Attila Fejér (D) https://orcid.org/0000-0002-2680-4723

Zoltán Nagy (D) https://orcid.org/0000-0002-0992-7123

\section{REFERENCES}

1. Mick S, Lapeyre M, Rouanet P, et al. Reachy, a 3D-printed human-like robotic arm as a testbed for human-robot control strategies. Front Neurorobot. 2019;13:1-65. https://doi.org/10.3389/fnbot.2019.00065

2. Mick S, Segas E, Dure L, et al. Shoulder kinematics plus contextual target information enable control of multiple distal joints of a simulated prosthetic arm and hand. J NeuroEngineering Rehabil. 2021;18(1):1-17. https://doi.org/10.1186/s12984-020-00793-0

3. González-Díaz I, Benois-Pineau J, Domenger J-P, de Rugy A. Perceptually-guided understanding of egocentric video content: Recognition of objects to grasp. In: Proceedings of the 2018 ACM on International Conference on Multimedia Retrieval. Association for Computing Machinery; 2018; New York, NY, USA:434-441. https://doi.org/10.1145/3206025.3206073

4. Lowe DG. Distinctive image features from scale-invariant keypoints. Int J Comput Vision. 2004;60:91-110.

5. Fejér A, Nagy Z, Benois-Pineau J, Szolgay P, de Rugy A, Domenger J. FPGA-based sift implementation for wearable computing. In: 2019 IEEE 22nd International Symposium on Design and Diagnostics of Electronic Circuits Systems (DDECS). Cluj-Napoca, Romania; 2019:1-4.

6. Rublee E, Rabaud V, Konolige K, Bradski G. Orb: an efficient alternative to sift or surf, Proceedings of the 2011 International Conference on Computer Vision. IEEE Computer Society; 2011; Washington, DC, USA:2564-2571. https://doi.org/10.1109/ICCV.2011.6126544

7. Bay H, Ess A, Tuytelaars T, Van Gool L. Speeded-up robust features (surf). Comput Vis Image Underst. 2008;110(3):346-359. https://doi. org/10.1016/j.cviu.2007.09.014

8. Rosten E, Drummond T. Machine learning for high-speed corner detection. In: Leonardis A, Bischof H, Pinz A, eds. Computer VisionECCV 2006. Berlin, Heidelberg: Springer Berlin Heidelberg; 2006:430-443.

9. Calonder M, Lepetit V, Strecha C, Fua P. Brief: binary robust independent elementary features. In: Proceedings of the 11th European Conference on Computer Vision: part IV. Springer-Verlag; 2010; Berlin, Heidelberg:778-792.

10. Karami E, Prasad S, Shehata MS. Image matching using sift, surf, BRIEF and ORB: performance comparison for distorted images. arXiv preprint arXiv:1710.02726; 2017.

11. Bradski G. The OpenCV Library. Dr Dobb's J Softw Tools. 2000;120:122-125.

12. Hess R. An open-source siftlibrary. In: Proceedings of the 18th ACM International Conference on Multimedia. ACM; 2010; New York, NY, USA:1493-1496. https://doi.org/10.1145/1873951.1874256

13. Li Z, Jia H, Zhang Y. Hartsift: a high-accuracy and real-time sift based on gpu. In: 2017 IEEE 23rd International Conference on Parallel and Distributed Systems (ICPADS). Shenzhen, China; 2017:135-142.

14. Björkman M, Bergström N, Kragic D. Detecting, segmenting and tracking unknown objects using multi-label mrf inference. Comput Vision Image Understand. 2014;118:111-127. https://doi.org/10.1016/j.cviu.2013.10.007

15. Doménech-Asensi G, Zapata-Pérez J, Ruiz-Merino R, et al. All-hardware sift implementation for real-time VGA images feature extraction. J Real-Time Image Process. 2020;17(2):371-382. https://doi.org/10.1007/s11554-018-0781-0

16. Rubio-Ibáñez P, Ruiz-Merino R, Doménech-Asensi G, et al. An all-hardware implementation of the subpixel refinement stage in sift algorithm. Int J Circuit Theory Appl. 2018;46(9):1690-1702. https://doi.org/10.1002/cta.2482

17. Vourvoulakis J, Kalomiros J, Lygouras J. FPGA accelerator for real-time sift matching with ransac support. Microprocessors Microsyst. 2017;49:105-116. https://doi.org/10.1016/j.micpro.2016.11.011

18. Vourvoulakis J, Kalomiros J, Lygouras J. Fully pipelined FPGA-based architecture for real-time sift extraction. Microprocessors Microsyst. 2016;40:53-73. https://doi.org/10.1016/j.micpro.2015.11.013

19. Chang L, Hernández-Palancar J, Sucar LE, Arias-Estrada M. FPGA-based detection of sift interest keypoints. Machine Vision Appl. 2013; 24(2):371-392. https://doi.org/10.1007/s00138-012-0430-8

20. Shao A, Wei-xian Q, Guo-hua G, Kai-li L. Real-time implementation of SIFT feature extraction algorithms in FPGA. In: 2015 International Conference on Optical Instruments and Technology: optoelectronic Imaging and Processing Technology, Vol. Proc. SPIE 9622. Beijing, China: SPIE; 2015:233-247. https://doi.org/10.1117/12.2190330

21. Rodríguez-Vázquez A, Domínguez-Castro R, Jiménez-Garrido F, et al. A CMOS vision system on-chip with multi-core, cellular sensoryprocessing front-end. In: Roska T, Baatar C, Porod W, eds. Cellular nanoscale sensory wave computing: Springer US; 2009:129-146. https://doi.org/10.1007/978-1-4419-1011-0_6

22. Suárez M, Brea VM, Fernández-Berni J, Carmona-Galán R, Cabello D, Rodríguez-Váquez A. Gaussian pyramid extraction with a cmos vision sensor. In: 2014 14th International Workshop on Cellular Nanoscale Networks and their Applications (CNNA). Notre Dame, USA; 2014:1-2.

23. Toshiba. SPS. http://www.toshiba-teli.co.jp/en/products/industrial/sps/sps.htm; 2019.

24. Daoud L, Latif MK, Jacinto HS, Rafla N. A fully pipelined FPGA accelerator for scale invariant feature transform keypoint descriptor matching. Microprocessors Microsyst. 2020;72:102919. https://doi.org/10.1016/j.micpro.2019.102919

25. Grasping in the wild. https://www.labri.fr/projet/AIV/dossierSiteRoBioVis/GraspingInTheWildV2.htm; 2016.

26. Vivado axi reference guide, 4th ed. https://www.xilinx.com/support/documentation/ip_documentation/axi_ref_guide/latest/ug1037vivado-axi-reference-guide.pdf; 2017. 
27. Vörösházi Z, Kiss A, Nagy Z, Szolgay P. Implementation of embedded emulated-digital cnn-um global analogic programming unit on FPGA and its application. Int J Circuit Theory Appl. 2008;36(5-6):589-603. https://doi.org/10.1002/cta.507

28. Xilinx. Vivado design tools. https://www.xilinx.com/products/design-tools/vivado.html; 2018.

How to cite this article: Fejér A, Nagy Z, Benois-Pineau J, Szolgay P, A de Rugy, Domenger J-P. Implementation of Scale Invariant Feature Transform detector on FPGA for low-power wearable devices for prostheses control. Int J Circ Theor Appl. 2021;49:2255-2273. https://doi.org/10.1002/cta.3025 